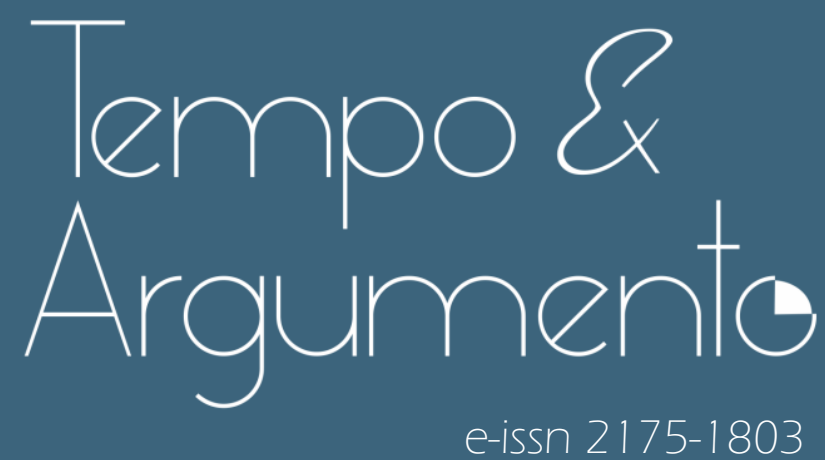

\title{
Género y currículum en disputa. Reflexiones sobre prácticas y saberes universitarios
}

- Luisina Bolla

Doctora en Filosofía por la Universidad Nacional de La Plata (UNLP).

Profesora en la Universidad Nacional de La Plata (UNLP/CONICET).

La Plata - ARGENTINA

memoria.fahce.unlp.edu.ar/perfiles/ 1688BollaL.html

luisinabolla@gmail.com

(iD) orcid.org/0000-0001-5860-8850

- Milagros María Rocha

Estudiante del Doctorado en Historia, Facultad de Humanidades y Cs.

de la Educación en la Universidad Nacional de La Plata (UNLP) y

Magister en Educación por la misma Universidad.

Profesora en la Universidad Nacional de La Plata (UNLP).

La Plata - ARGENTINA

memoria.fahce.unlp.edu.ar/perfiles/ 1 160RochaM.html

milagrosmrocha@gmail.com

(iD) orcid.org/0000-0002-5361-0211

Para citar este articulo:

BOLLA, Luisina; ROCHA, Milagros. Género y currículum en disputa. Reflexiones sobre prácticas y saberes universitarios. Tempo e Argumento, Florianópolis, v.

12, n. 30, e0207, maio/ago. 2020

doi http://dx.doi.org/10.5965/2175180312302020e0207

Recebido: 30/08/2019

Aprovado: 22/03/2020 


\title{
Género y currículum en disputa. Reflexiones sobre prácticas y saberes universitarios
}

\begin{abstract}
Resumen
El presente artículo reflexiona sobre tres ejes: el primero alude al currículum universitario en tanto artefacto de género (SILVA, 1998) o tecnología de género (DE LAURETIS, 2000). A partir del análisis de programas correspondientes al Profesorado en Historia de la Universidad Nacional de La Plata (UNLP) mostramos cómo los contenidos relacionados a género y mujeres, en las materias obligatorias de la carrera, comienzan a visibilizarse con mayor vigor en 2016 (ROCHA, 2019). No obstante, si bien estos contenidos encuentran por donde "filtrarse", notamos que esto ocurre en espacios curriculares optativos, de manera sistemática a partir de 2005. El segundo eje analiza lo que denominamos el currículum en disputa a partir del análisis de ciertos porcentajes, por cierto, mínimos, que referencian a las mujeres como autoras de la bibliografía que se lee en los primeros años de las carreras de la Facultad de Humanidades y Ciencias de la Educación (UNLP). Para ello, realizamos un análisis exploratorio de asignaturas introductorias de la carrera de Filosofía que muestran la dialéctica presencia/ausencia de mujeres en los programas. Tercero y último, retomamos aportes de las epistemologías feministas para problematizar las formas en que se incluyen y se enseñan los contenidos vinculados al género y los feminismos en la Universidad.
\end{abstract}

Palabras clave: Feminismos. Género. Ciencia. Currículum. Universidad.

\section{Gender and curriculum trouble. Reflections on university practices and knowledge}

\begin{abstract}
This article reflects on three axes: the first refers to the university curriculum as a gender artifact (SILVA, 1998) or gender technology (DE LAURETIS, 2000). From the analysis of programs corresponding to History career of the Universidad Nacional de La Plata (UNLP), we show how the contents related to gender and women, in the compulsory subjects of the degree, began to become more visible in 2016 (ROCHA, 2019). However, although these contents find where to "filter", we note that this occurs in optional curricular spaces, systematically from 2005. The second axe focus on what we call curriculum trouble from the analysis of certain percentages, for certain minimums, which refer to women as authors of the bibliography that is read in the early years of the careers of the Faculty of Humanities and Educational Sciences (UNLP). In order to do this, we develop an exploratory analysis of introductory subjects of the Philosophy degree that show the dialectic presence/absence of women in the programs. Third and final, we resume contributions from feminist epistemologies to problematize the ways in which contents related to gender and feminisms are included and taught at the University.
\end{abstract}

Keywords: Feminisms. Gender. Science. Curriculum. University. 


\section{Premisas y fundamentos epistémicos de la investigación}

En Argentina, la sanción de la Ley 26.150 de Educación Sexual Integral (ESI) en el año 2006 contribuyó a ubicar en el centro de la escena pública el debate en torno a la necesidad de una enseñanza crítica y no sexista. Recordemos que el Programa Nacional de Educación Sexual Integral establece que "todos los educandos tienen derecho a recibir educación sexual integral en los establecimientos educativos públicos, de gestión estatal y privada de las jurisdicciones nacional, provincial, de la Ciudad Autónoma de Buenos Aires y municipal" (ARGENTINA, 2006, art. 1). Junto con este objetivo general, la Ley ESI innova al proponer la introducción de una perspectiva de género transversal a las diferentes materias, que quite a la educación sexual del terreno circunscripto de la biología y que permita su problematización en las diferentes áreas. Este proceso, signado por importantes avances, pero también con resistencias de parte de sectores religiosos y conservadores, logró de todos modos instalar la discusión en torno a una enseñanza no sexista y transversal.

Sin embargo, el debate impactó de modo más significativo en los sectores primarios y medios que en los sectores universitarios. Extrapolando la observación de Mariela Coudannes -sobre la creencia, bastante aceptada, de que sólo con "saber historia" alcanza para enseñarla y en esa línea, no es necesario reflexionar sobre qué, para qué y cómo enseñar- la autora expresa que, si bien "mucho se ha escrito sobre la transposición de contenidos en la escuela secundaria argentina, menos se ha debatido en las instituciones universitarias respecto de la enseñanza en el propio nivel" (COUDANNES, 2010, p. 976).

En esta clave, el presente artículo inicia una reflexión acerca del quéy para qué, es decir, qué contenidos y bibliografía se selecciona para abordar la perspectiva de género en el ámbito universitario, que implica también una indagación acerca de las condiciones de producción, esto es, de los sesgos y subtextos que estructuran esos discursos. Finalmente, propondremos una reflexión sobre el cómo se introducen los temas vinculados a feminismos y género, abordando las dificultades y desafíos de su incorporación en espacios generalmente optativos y/o su inclusión como temas especiales. 
Con el objetivo de realizar un aporte al debate sobre la enseñanza crítica y no sexista en el plano universitario, proponemos un análisis de programas de cátedra correspondientes al Profesorado en Historia de la Universidad Nacional de La Plata y un estudio exploratorio de algunas asignaturas de Introducción a la Filosofía en diferentes Universidades Nacionales. En este marco el objetivo inicial es explorar la presencia o ausencia de las mujeres en la enseñanza de la Historia y Filosofía y, a su vez, evaluar si tal dialéctica presencia/ausencia trae como correlato una problematización en clave feminista y de género. ${ }^{1}$ Retomamos para ello el espíritu crítico que la Ley ESI impulsa respecto de la educación sexual, en particular, su propuesta en términos de obligatoriedad y transversalidad, para repensar la educación universitaria en sentido amplio a través del prisma de las teorías feministas y de los estudios de género.

Si bien la herramienta de acceso de la investigación serán los programas de estudio de las carreras universitarias, es importante aclarar que, aunque dichos documentos poseen una información valiosa,

no agota[n] la explicación de lo que la práctica efectivamente es, se parte de la afirmación - sustentada en teoría e investigación que define a estos discursos como reguladores de la acción y enmarcadores de un sistema de clasificación social y pedagógica que determina qué le corresponde al magisterio y el "mapa del saber" que se le adscribe en la formación. (DAVINI, 1998, p. 189)

La cita de Davini nos permite explicitar el alcance que habilita este tipo de documentos sabiendo, como expresa la autora, que éstos por sí mismos no agotan el estudio de la puesta en acción en la enseñanza. Partiendo de este reparo, argumentamos entonces la validez del análisis de estos escritos como discursos reguladores, entendiendo que en el currículum universitario se aloja "la mayor y más variada carga de saberes académicos representativos de los campos de conocimiento con alto grado de especialización y proximidad respecto del mundo del trabajo para el que forman" (COSCARELLI, 2014, p. 99100).

\footnotetext{
'Si bien ambos términos no son, por supuesto, sinónimos, en este trabajo apostaremos por una integración de estas dos perspectivas, según justificaremos a continuación (cf. Infra, apartado "Estudios de género y feminismos").
} 
Sustentamos esta investigación a partir de la observación de dos de los componentes del programa (FELDMAN, 2010): contenidos y bibliografía. Nos proponemos indagar en un doble sentido: por un lado, explorar de qué modo ingresan temas vinculados a mujeres y feminismos en los contenidos de las carreras, y por otro, ahondar en la bibliografía seleccionada y citada. Puntualmente nos interesa analizar el porcentaje de representatividad de mujeres-autoras para luego - en la segunda parte del artículo - explorar filosófica e históricamente las consecuencias de estos resultados. En tal sentido, el análisis situado de los programas de estudio permitirá movilizar una reflexión más general sobre la crítica feminista de la producción de conocimientos científicos y filosóficos, esto es, evaluar sus alcances y los desafíos aún abiertos.

\section{Estudios de género y feminismos}

Antes de proseguir, consideramos necesario realizar una breve advertencia sobre el uso de las categorías de análisis. Es sabido que los conceptos de "género" y "feminismo" provienen de tradiciones intelectuales y políticas no siempre convergentes. En Argentina, el término "género" se ha impuesto progresivamente en el campo académico y actualmente constituye la designación más corriente.

Recordemos brevemente que desde el retorno de la democracia en la década del '80, comenzaron a ganar espacio en las universidades de nuestro país los programas de "Estudios de la Mujer". A fines de la década del "90 y durante los primeros años del milenio, estos comenzaron a denominarse "Estudios de Género". Esta designación, si bien con excepciones, tendió a adoptarse en las cátedras, proyectos, laboratorios y centros. ${ }^{2}$ En tal sentido, la denominación "estudios de género" fue imponiéndose progresivamente sobre el adjetivo "de

\footnotetext{
${ }^{2}$ Un ejemplo de dicho proceso fue la creación del Instituto de Investigaciones en Estudios de Género (IIEGE) de la Facultad de Filosofía y Letras de la Universidad de Buenos Aires, en el año 1997, dirigido desde el año 2000 por la historiadora Dora Barrancos. El antecedente del IIEGE en dicha institución fue el Área Interdisciplinaria de Estudios de la Mujer (AIEM) fundada en julio de 1992. En la Universidad Nacional de La Plata, en el año 2006 se creó el Centro Interdisciplinario de Investigaciones en Género (CInIG), aprobado formalmente en el año 2007 por la Secretaría de Ciencia y Técnica de la UNLP (res. no 49/07 del Consejo Superior). Su primer director fue el Dr. José Amícola y su co-directora la Dra. María Luisa Femenías.
} 
mujeres" y "feminista", proceso que parece coincidir con lo ocurrido en otros países del Cono Sur (CISNE, 2016).

Tal utilización del concepto de "género" pareció obedecer a un interés estratégico, dado que permitía el ingreso de las reflexiones "feministas" y "de las mujeres" en la universidad bajo una designación que se presentaba como neutral y que no remitía a los sentidos políticos asociados a aquellas. Como hemos sostenido en otro trabajo, en consonancia con investigaciones recientes, de algún modo "estudios de género" era más aceptable para la objetividad académica que "estudios feministas" o "de las mujeres" (BOLLA, 2016). En parte, debido a que su transversalidad no cuestionaba las divisiones de las disciplinas ya constituidas (BARZANI, 2015). En parte, porque se prestaba más fácilmente a su abstracción, como ocurre cuando es utilizado de forma aislada, sin volverse coextensivo con las relaciones de clase y raza (CISNE, 2016). Veremos que estas tensiones se mantienen parcialmente en la actualidad.

En el ámbito donde nos desempeñamos y en base al cual elaboramos las reflexiones aquí presentadas, la Universidad Nacional de La Plata (Argentina), comienza sin embargo a producirse una cierta transformación respecto de lo acaecido durante la década del "90 y principios de los años 2000. Durante los últimos años, el término "feminismo" vuelve a ingresar en los espacios académicos, en general, a la par de la designación "género". De este modo, la práctica aproxima aquello que, en muchas discusiones teóricas, puede presentarse como un antagonismo. ${ }^{3}$ Siguiendo esta tendencia de nuestro campo académico, a lo largo de estas líneas utilizaremos ambas denominaciones.

\footnotetext{
3 A modo de ejemplos, podemos referir la creación de una cátedra optativa en la Facultad de Humanidades y Ciencias de la Educación, denominada "Introducción a la teoría feminista y de género" (desde el año 2016), optativa para todas las carreras de grado de dicha Facultad, o la revista asociada al Centro Interdisciplinario de Investigaciones en Género, fundada en el año 2017: Descentrada. Revista Interdisciplinaria de Feminismos y Género. Entendemos que el diálogo entre ambos campos de producción teórica encuentra un basamento: si las teorías feministas visibilizan una estructura de dominación basada en el sexo-género, es decir, una matriz que constituye grupos sociales jerarquizados y binarios ("varones" y "mujeres"), los estudios de género pueden insistir en la denuncia del orden heteronormativo que funda tal estructura de dominación sexista (comúnmente denominada "patriarcal" o "androcéntrica") mostrando también los efectos opresivos que sufren todas aquellas personas cuyas identidades y/o sexualidades se alejan de la norma binaria. En ambos casos, el supuesto compartido debería ser el carácter social (político) de las categorías de sexo-género, lo que evita tanto el escollo del biologicismo (que atraviesa diversas tendencias feministas aún en la actualidad) como la ahistorización o eventual omisión del orden patriarcal que produce y regula las categorías de sexo-género.
} 
Con el objetivo de profundizar estas reflexiones y de comprender tales procesos en su carácter situado, desarrollamos a continuación nuestra investigación empírica, que permitirá al final de este artículo revisitar algunos de estos interrogantes desde una perspectiva crítica. Veremos que los límites porosos entre las regiones y conceptos siguen vigentes y que sus consecuencias distan mucho de constituir meros problemas nominales. ¿Cómo se desarrolla este vínculo entre la "perspectiva de género" y las "teorías feministas”? ¿Qué consecuencias trae aparejada su institucionalización creciente en las universidades? Y en particular, ¿qué desafíos encuentra la transversalización de estas perspectivas?

\section{Género y feminismos en los programas de Historia}

En la carrera de Historia, si bien hallamos indicios de una visibilidad creciente referida a temas de género y feminismos, lo cierto es que los espacios curriculares en donde emergen se circunscriben, principalmente, a materias optativas de la carrera. ${ }^{4}$ En términos generales en el caso de las materias obligatorias podemos señalar que hay, en los últimos años, un alto porcentaje de cátedras que se están preguntando por la participación de las mujeres, el rol que éstas cumplen, en conexión con las construcciones socioculturales. Asimismo, indicamos que la bibliografía que visibiliza a las mujeres puede cumplir con una función visualizadora, aunque no necesariamente se trate de una perspectiva feminista o de género, es decir, que re-interprete esos roles de género analizando su jerarquización.

En este sentido mencionamos que, en el marco del Plan de Estudios '93, cinco asignaturas (sobre un total de doce materias correspondientes al bloque troncal obligatorio) incluyen estas temáticas, ${ }^{5}$ mientras que luego de aprobarse el Plan de Estudios vigente (2011) otras seis materias se suman e incorporan bibliografía afín (cabe destacar que con el cambio de Plan el número de las

\footnotetext{
${ }^{4}$ Por caso mencionamos que desde el 2005 en adelante, la profesora Adriana Valobra dicta de manera sistemática una materia optativa en donde aborda estos temas como eje estructurante. En 2017, pioneramente, dicta una materia que pone a debate las masculinidades (Programa disponible: http://www.memoria.fahce.unlp.edu.ar/programas/pp.10702/pp.10702.pdf)

${ }^{5}$ Esta información se obtiene a partir de un formulario virtual enviado a docentes de la carrera.
} 
materias obligatorias aumenta a quince).Asimismo, señalamos que dentro de este último agrupamiento el año 2016 resulta una fecha en donde varias de las seis cátedras aludidas incluyen estos temasen sus programas (ROCHA, 2019). Por tanto, en algunos casos, dichas incorporaciones resultan más recientes. Más allá de identificar esto nos preguntamos el lugar que ocupan estos temas dentro de los programas: ¿qué voces y sujetxs del pasado se estudian? En esta línea Débora d'Antonio expresa:

El estudio del género y la sexualidad se ha constituido en un campo aparte, en una suerte de subespecialidad que no influencia el corazón de la producción histórica en los terrenos fundamentales de la economía, la política, la cultura o la historia social. En los cursos de grado impartidos en las universidades nacionales, la bibliografía que aborda esta relación es prácticamente inexistente o en el mejor de los casos sólo forma parte marginal del currículum académico. (D 'ANTONIO, 2012-2013, p. 10)

El planteo de la autora nos permite referir dos cuestiones, la primera vinculada a cuestiones historiográficas, es decir, al argumento donde se sostiene que este tipo de investigaciones todavía no ha "influenciado el corazón de la producción histórica”. El otro aspecto por destacar es el lugar que se le otorga, en la enseñanza, a estos temas. Como señalan Elizalde, Felitti y Queirolo (2009) el género continúa siendo una categoría explicativa relegada para estudiar y comprender la historia. Entonces, ¿qué categorías analíticas tienen y continúan teniendo legitimidad en nuestras carreras? ¿Por qué todavía la categoría de género resulta "lo otro", "lo especial”?

El relevo de programas de las materias obligatorias 2019 presenta otro escenario. La categoría de género se hace más evidente desde el enunciado de los propios contenidos, además de la bibliografía. Esta situación dista respecto del análisis realizado a partir de los programas 2016/2017. En ese sentido hallamos varias diferencias, por un lado, dicha categoría se hace más visible en 2019 incorporándose en varias unidades de los programas de algunas de estas materias, esto equivale además en un acrecentamiento de bibliografía que aborda tales temas. Por otro, se suma otra materia (a esas seis referidas del Plan 2011) que explicita la inclusión de estos contenidos en varias unidades del programa. En suma, historizar brevemente este recorrido permite plantear no 
sólo un alto porcentaje de materias que están alojando estos temas y perspectivas, sino también presenta la idea de un currículum en movimiento y transformación. Un currículum que comienza a descolonizarse de esa impronta masculina.

\section{El currículum en disputa}

Tempranamente, diferentes investigadoras provenientes del ámbito de las epistemologías feministas mostraron que la ciencia - y, agregamos también, la filosofía - son construcciones sociales, atravesadas por sesgos y subtextos patriarcales (FOX KELLER, 1991; HARAWAY, 1995). ${ }^{6}$ Ahora bien, el currículum también es una construcción social (SILVA, 1999). Éste no resulta neutral ni aséptico, justamente detrás de éste hay todo un conjunto de decisiones que se toman de índole epistemológica, pedagógica, didáctica. A partir de los resultados de nuestras investigaciones en la carrera de Historia (ROCHA, 2019), identificamos un currículum que en los últimos años comienza a flexibilizarse.

Dentro de las decisiones que se toman para confeccionar y delimitar el programa de una materia, aparece otro aspecto importante como la bibliografía. Nos preguntamos, por ejemplo, qué autores y autoras leemos en nuestras carreras, quiénes fueron o son los que poseen la palabra "autorizada", y en vinculación a un planteo en clave de "saberes situados" (HARAWAY, 1988) desde dónde se piensa y plantea la teoría que estudiamos.

Durante el mes de mayo de 2018 la agrupación estudiantil "La Jauretche" nos convocó a una charla para debatir sobre "Mujeres y saberes hegemónicos"; actividad que se realizó en la Facultad de Humanidades y Ciencias de la Educación (UNLP). La propuesta se generó en función de los llamativos porcentajes relevados por la propia organización, en cuanto a la cantidad de autoras mujeres que se leen en los primeros años en las carreras de nuestra

\footnotetext{
El giro historicista kuhniano habilitó una senda de indagación propicia para las diversas epistemólogas feministas, que recusaron definitivamente la ilusión de un conocimiento neutro, aséptico y cuestionaron la ilusión del punto cero. Dado que no exploraremos las divergencias entre cada una de las propuestas, baste recordar que tampoco constituyen un conjunto homogéneo, sino que se distinguen diferentes tendencias, como oportunamente mostró Sandra Harding (1996). Más adelante retomaremos algunas de estas discusiones.
} 
Facultad. Su indagación arrojó resultados contundentes: las carreras de Historia y Filosofía, 87\% varones, 13\% mujeres; Educación Física y Letras, 78\% varones, 22\% mujeres; Traductorado de inglés, 74\% varones y 26\% mujeres; Sociología, $83,4 \%$ varones, $16,6 \%$ mujeres.

Si tomamos de referencia, por caso, algunas de las materias introductorias de las carreras (Introducción a la Historia, Introducción a la Filosofía, Sociología General, entre otras) podemos señalar que las mismas se dirigen, principalmente, a ubicar en tiempo y espacio el origen del objeto de estudio. Es decir, indagan en los antecedentes e historizan el surgimiento de la Historia, Filosofía, Sociología, etc. Si precisamos este análisis podemos ver cómo los "padres" de estas disciplinas, o quienes personifican esos "orígenes" fundantes, son principalmente varones y europeos.

Por otro lado, las lecturas que reinterpretan a estos referentes ("bibliografía secundaria") también remiten en la mayoría de los casos a autores varones. Frente a este análisis y los porcentajes relevados, bien vale preguntarse por el rol de las mujeres en el conocimiento y el reconocimiento de éstas en las ciencias y en la filosofía. La preocupación de esta agrupación levanta una disputa que si bien no es nueva pone de relieve el currículum como "artefacto" o "tecnología de género" (DE LAURETIS, 2000; SILVA, 1999) en donde no sólo se corporizan sino también se producen relaciones de género. Nos preguntamos entonces en qué medida este currículum colonizado por la masculinidad (europea) produce y reproduce tales hegemonías.

A fines de obtener una visión más general sobre este problema, decidimos tomar como ejemplo la carrera de Filosofía, una de las que cuenta con menor representación "femenina" en los programas. Nos propusimos cotejar los porcentajes relevados por la agrupación estudiantil en la Facultad de Humanidades y Ciencias de la Educación de La Plata, con el caso de otras asignaturas afines en diferentes universidades nacionales argentinas. ${ }^{7}$ Si bien se trata de un estudio exploratorio, nos permitió comprobar que otras materias

\footnotetext{
A la fecha, por motivos ajenos a ambas partes, no hemos logrado tener acceso a los registros que sustentaron las estadísticas de la agrupación estudiantil. No obstante, queremos destacar la buena disposición de los referentes de dicha agrupación ante nuestras preguntas y su generosidad para con el tema.
} 
introductorias de Filosofía muestran la misma tendencia. Un primer análisis abordó los programas de nueve universidades nacionales: Universidad Nacional de Mar del Plata, Universidad de Buenos Aires, Universidad Nacional de Córdoba, Universidad Nacional del Sur, Universidad Nacional de Santiago del Estero, Universidad Nacional de Cuyo, Universidad Nacional de Salta, Universidad Nacional del Litoral y la propia Universidad Nacional de La Plata. ${ }^{8}$

Luego del análisis, encontramos un muy reducido número de textos escritos por mujeres como parte de la bibliografía obligatoria, que van del 0 al tope máximo de 9\% sobre el total de los textos (este último porcentaje máximo, en la Universidad Nacional de La Plata). El porcentaje total obtenido es de 3,25\% de mujeres en la bibliografía obligatoria citada. En algunos casos, este porcentaje aumenta al considerar la bibliografía complementaria. Al respecto, observamos que suele tratarse de textos escritos por las profesoras a cargo de la materia, o bien de profesoras que integran el equipo docente de la cátedra.

Es decir, que si bien encontramos algunas excepciones de filósofas citadas (Agnes Heller, Hannah Arendt, Cristina Lafont), en general el porcentaje de representatividad de mujeres en los programas aumenta ligeramente con la inclusión de textos escritos por las titulares de esas cátedras (en los casos en que son mujeres quienes están a cargo de dichas cátedras).

Sin embargo, es preciso señalar que, en la mayoría de los casos, no se trata de autoras que abordan teóricamente el feminismo ni de textos que problematizan la temática de género, lo cual nos conduce a otro interrogante: ¿Es suficiente la presencia de mujeres-autoras para transformar estas disciplinas? Si bien, obviamente, la paridad constituye un horizonte deseable por el cual debemos seguir luchando, pareciera que tampoco alcanza sólo con aumentar el número de autoras mujeres, si eso redunda en una invisibilización de las problemáticas de opresión según sexo-género.

\footnotetext{
B Esta primera muestra, a fines exploratorios, tomó como objeto los programas disponibles al año 2018 que se encontraban disponibles online y deberá ser ampliada en futuras investigaciones. En todos los casos, corresponden a materias introductorias correspondientes al bloque obligatorio del primer año, de carreras de Lic. y Prof. en Filosofía, en Universidades Nacionales de Argentina. La selección intentó respetar un principio de pluralidad territorial, focalizando diferentes regiones. Para tener una referencia, la carrera de Lic. en Filosofía se encuentra en 21 universidades nacionales argentinas; la muestra inicial aquí presentada representa casi la mitad de dicho universo.
} 
Asimismo, advertimos que estas estadísticas corren con la desventaja de dicotomizar el análisis, ya que una podría preguntarse también cuántxs autorxs trans/travestis se leen. Sin embargo, al mismo tiempo, no dejan de aportar información importante para repensar lo que ocurre en el espacio universitario actual. Otro dato por mencionar es que, entre todas las carreras relevadas por los análisis de la agrupación estudiantil mencionada, Historia y Filosofía resultan las carreras con menor porcentaje de autoras.

Refiriéndose puntualmente al campo universitario, Graciela Morgade ha sostenido que las Universidades Públicas argentinas resultan, todavía, "territorios patriarcales”. A propósito del lugar de la mujer en la ciencia Morgade afirma que

la incorporación de las mujeres no ha derivado aún en una crítica epistemológica de las ciencias, en todas sus expresiones, tanto en las modalidades de construcción del conocimiento como en la lengua que se emplea en ese proceso y las categorías teóricas derivadas. (MORGADE, 2018, p. 33)

Siguiendo a esta autora nos preguntamos por qué esta crítica epistemológica, que ha logrado cuestionar los procedimientos y métodos científicos, parece demorarse cuando se trata de explorar esos artefactos, especialmente el currículum.

\section{Una epistemología radical}

En el intento por comprender estas cuestiones, es de interés retomar la propuesta de la filósofa Diana Maffía (2007) que ha agrupado las posiciones de las epistemologías feministas según sus diversos abordajes conceptuales. Intentaremos extrapolar estas observaciones críticas para aplicarlasal análisis de los programas universitarios, desplazándonos de la actividad y ejercicio de la ciencia básica al plano de su enseñanza. Mostraremos que los aportes de las epistemologías feministas proporcionan claves metodológicas para repensar la enseñanza de la ciencia y de la filosofía en el nivel universitario.

Siguiendo la clasificación de las epistemologías feministas que ofrece Maffía (2007), por un lado, encontramos posiciones que buscan recuperar las contribuciones de mujeres en la ciencia, analizando la invisibilización histórica de 
las mujeres del ámbito científico. Junto a estas, encontramos posiciones que complementan el enfoque anterior, estudiando los modos en que históricamente las mujeres participaron en instituciones científicas (participación signada por un desigual acceso a recursos, medios de producción y menor estatus dentro de las profesiones). Otras investigaciones tendieron a analizar el modo en que las diversas ciencias definieron a las mujeres, en particular, en los ámbitos de la medicina y de la biología; lo que podríamos denominar siguiendo a Amorós (1991) el estudio de las mujeres quaobjetos de los discursos académicos y científicos. Finalmente, sostiene Maffía, encontramos otras posiciones que visibilizan el androcentrismoen el que se funda la ciencia moderna, explorando las distorsiones que acarrea la ausencia de las mujeres en las formas del conocimiento científico y que incluye también la expulsión de cualidades consideradas "femeninas" del ámbito de la ciencia.

La clasificación de Maffía resulta interesante en la medida en que invita a entramar estas dimensiones, de modo integral, en vistas a una epistemología (y de modo más general, a una "crítica") feminista que no permanezca en el plano de los casos individuales o "excepciones" sino que problematice también los procesos y normas considerados válidos para la legitimación científica. Como señala Maffía, no se trata sólo de "abrir la ciencia" a las mujeres o de “incorporarlas", según señalaba críticamente Morgade. Si bien es condición necesaria, ello no es suficiente. Hace falta también transformarla ciencia, sus valores, sus métodos, sus prácticas, desde el feminismo, lo que Maffía denomina "punto de vista radical" en la epistemología feminista:

En lo personal, creo que la ciencia debe considerarse en su doble aspecto de proceso y producto, y que ambos son sexistas. Hay un sexismo, que ha sido brillantemente señalado por muchas epistemólogas, en las teorías científicas (producto); hay otro en la composición y exigencias de pertenencia y méritos, en las comunidades científicas (proceso). El desafío del feminismo consiste en mostrar el vínculo entre ambos, y señalar que una mayor apertura en las comunidades conducirá, si no a un cambio radical en el conocimiento, al menos a una ciencia menos sesgada. (MAFFÍA, 2007, p. 65)

Las observaciones de Maffía sobre la ciencia nos permiten repensar nuestro análisis del currículum universitario. En efecto, no se trata simplemente 
de "incluir" mujeres en los programas para alcanzar una igualdad formal o paritaria y así obtener "un currículum menos sesgado". El desafío es transformar los contenidos, interpelarlos desde el horizonte crítico de las teorías feministas y de género. ${ }^{9}$

A nuestro juicio, ello implica entramar las cuatro dimensiones que mencionamos anteriormente: 1) visibilizar el rol de las mujeres como productoras y agentes de la ciencia y la filosofía, esto es, qua sujetos de conocimientos. Supone tanto recuperar genealogías invisibles como subrayar la subsistencia de desigualdades en cuanto a acceso y participación en los ámbitos científicos y académicos; 2) analizar lo que las diferentes disciplinas han dicho sobre las mujeres y sexualidades disidentes, qua objetos, es decir, problematizar el canon; y finalmente 3) visibilizar el androcentrismo que atraviesa las disciplinas desde su propio surgimiento para contribuir al ejercicio crítico de su desnaturalización, problematización y finalmente, transformación. Tanto en el caso de la ciencia como en el de la filosofía, la exclusión de la mitad numérica de la especie (minoritaria en términos de poder, al decir de Colette Guillaumin) "necesariamente tiene que haber tenido (y aún tiene) consecuencias, cuanto menos, gnoseológicas y éticas distorsionantes en el discurso filosófico" [y científico] (FEMENÍAS, 2015, p. 89).

Otro dato relevante por destacar es que buena parte de los relevamientos estadísticos nacen a partir de inquietudes estudiantiles. Estxs sujetxs del desarrollo curricular (DE ALBA, 1998) son quienes vivencian cotidianamente el currículum, y en este caso, le imprimen otro significado. El ejercicio crítico reflexivo por parte de lxs estudiantes aporta a seguir identificando lógicas construidas y sedimentadas. La acción misma de construir esas estadísticas abona a ese punto de vista radical del que hablaba Maffía, al preguntarse no sólo por la autoría de esas lecturas sino también por las propias teorías construidas en función de quién escribe. En definitiva, una gimnasia que articula aspectos de producto y proceso.

\footnotetext{
Lo que, como mostramos al final de este artículo, implica necesariamente la transversalización de las perspectivas feministas y de género para evitar la sectorización de los debates. Caso contrario, puede ocurrir que estos se vuelvan "tolerables", es decir, que se acepten parcialmente en el modo de "temas otros" sin afectar o modificar los supuestos de base de las disciplinas y contenidos tradicionales.
} 
Ahora bien, la pregunta por el quién, es decir, por la autoría de los textos del currículum, evidentemente presenta varias dimensiones que no se reducen únicamente al "patriarcado". Lo que Maffía (2007, p. 64) denomina "androcentrismo epistémico" representa el punto de vista del varón que es además blanco, adulto, propietario, capaz. En tal sentido, consideramos pertinente preguntarnos qué feminismos estudiamos en la universidad. Dado que la respuesta a este interrogante excede los límites propuestos en este trabajo, nos limitaremos a señalar que la elaboración de un punto de vista epistemológico radical debería entrecruzar la perspectiva de análisis feminista con un abordaje que visibilice otros importantes sesgos de la ciencia, en particular, el racismo y el eurocentrismo.

De este modo, una aproximación feminista desde América Latina también deberá asumir el desafío de problematizar qué mujeres y qué feminismos aparecen o se ocultan en el canon. Vale la pena señalar que actualmente algunas cátedras optativas dentro de la UNLP presentan programas que incorporan de modo vertebral esta perspectiva de género y feminista, articulándola con una dimensión crítica de la colonialidad. ${ }^{10}$

Siguiendo a María Luisa Femenías, un desafío contemporáneo consiste en "la necesidad de que las mujeres contribuyamos con análisis específicos a fin de desentrañar cómo las narrativas hegemónicas de los 'varones del tercer mundo' y ciertos feminismos del 'primero' refuerzan lo que, a veces, se ha denominado “doble subalternidad”' (FEMENÍAS, 2009, p. 43).Para ello, entendemos que es necesario apostar por la (re)construcción de genealogías situadas que eviten tanto el androcentrismo como el eurocentrismo que muchas veces transversalizan los Planes de Estudio de nuestras universidades.

\section{Trans-formar el "tema especial" en transversalidad}

Hasta aquí, hemos visto que existe una dialéctica entre presencia y ausencia de las mujeres en los programas de las carreras. Hemos mostrado

\footnotetext{
${ }^{10}$ Desde el año 2016, por ejemplo, la Facultad de Humanidades y Ciencias de la Educación ofrece una materia optativa para todas las carreras denominada "Introducción a teoría feminista, estudios de género y sexualidades" que problematiza las genealogías feministas, contextualizando las "olas" e incluyendo la "colonialidad e interseccionalidad" como claves de un "enfoque transversal e interdisciplinario" (PROGRAMA, 2017, p. 4).
} 
también que, en los últimos años han ingresado con mayor vigor estos contenidos, lo que nos permite observar un currículum construido desde una masculinidad hegemónica que se produjo y reprodujo pero que de un tiempo a esta parte se encuentra en proceso de "trans-formación”. Luego de analizar los programas de las materias, entendemos que el desafío será pensar la superación de los dualismos binarios y de los posicionamientos dicotómicos. Apostamos por una trans-formación en términos de lo que Castro Gómez repone sobre el significado etimológico de la raíz trans:

tiene la misma raíz etimológica que la palabra "tres" y significa, por ello, la transgresión del dos, es decir, aquello que va más allá de los pares binarios que marcaron el devenir del pensamiento occidental de la modernidad: naturaleza/cultura, mente/cuerpo, sujeto/objeto, mente/espíritu, razón/sensación, unidad/diversidad, civilización/barbarie. La transdisciplinariedad busca cambiar esta lógica exclusiva ("esto o aquello") por una lógica inclusiva ("esto y aquello”). (CASTRO GÓMEZ, 2015, p. 81)

Planteamos la idea de pensar una trans-formación para que los estudios de género y mujeres, como su articulación con la crítica del racismo y del eurocentrismo, no sean "lo aquello", sino algo que pueda integrarse en la formación como parte de, "esto y aquello", superando de esta manera posicionamientos que lo presentan como clase o tema "especial", reforzando "lo aquello", así como los espacios donde se vuelven ausentes.

Como expresan Abate y Orellano (2015, p. 5), "el escenario actual requiere el despliegue de políticas educativas que reinterpreten el vínculo de la Universidad con la sociedad atendiendo a las demandas sociales y de formación”. De ahí el aporte de echar luz sobre ciertas construcciones devenidas en marginales $u$ omitidas, en pos de trans-formar y estudiar desde una mirada que integre un "espacio social generizado no homogéneo" (HARAWAY, 1995, p. 336). En este sentido nos parece importante retomar la categoría de “justicia curricular", utilizada por Robert William Connell. Connell sostiene que:

para garantizar la justicia social no sirven los currícula de guetos. Los currícula separados-y-diferentes tienen cierto atractivo, pero dejan intacto el currículum hegemónico en vigor. La justicia social requiere cambiar de punto de partida para reconstruir la corriente principal, de forma que encarne los intereses de las personas menos favorecidas. (CONNELL, 2009, p. 2) 
La estrategia de inclusión de ciertos temas bajo la modalidad de la clase especial corre el riesgo de no interrogar el canon vigente al mantener ciertos temas como "diferentes" o "marcados". Es decir que, paradójicamente, puede reforzar el carácter hegemónico del currículum que intentaría cuestionar. En palabras de Lopes Louro (2019, p. 3), el "carácter excepcional de ese momento pedagógico refuerza, una vez más, su significado de diferente y de extraño." Se trata, por el contrario, de mostrar cómo y por qué ciertos temas, sujetos y teorías han sido construidos como "diferentes", lo que implica -correlativamente - un cuestionamiento de aquello que se construye como "lo igual", término no marcado, punto cero o falso universal.

De ahí el desafío y apuesta por la trans-versalización de la perspectiva de género en el ámbito universitario. Sin embargo, todavía encontramos (y vivenciamos) ciertas resistencias a dicha transversalización. ${ }^{11}$ Más allá de los compromisos éticos y políticos en juego, la sectorización (o guetificación) de muchos debates se combina actualmente con la lógica de especialización fomentada por los sistemas científicos y académicos. Si bien, evidentemente, fue necesario constituir un campo de estudios autónomo ("estudios de la Mujer", luego predominantemente "de Género", actualmente quizás "feministas y de género") entendemos que un desafío actual supone complementar los estudios específicos con una transversalización efectiva de tales investigaciones. Es decir, que enfrentamos el desafío de trabajar para que las investigaciones feministas se integren en una problemática global (MATHIEU, 2013), lo que siguiendo a Maffía hemos denominado una crítica epistemológica radical. Mediante una comprensión dialéctica de este proceso podremos evitar que ciertas teorías o problemas queden relegados al papel del "tema especial" o "tema-otro", reafirmando así - involuntariamente - la lógica del pensamiento "Uno" que, a lo largo de estas líneas, hemos intentado problematizar.

\footnotetext{
11 Por mencionar una experiencia reciente, en el año 2018 presentamos en las X Jornadas de Sociología de nuestra Facultad una ponencia titulada: "Des-pensar para seguir pensando: saberes-¿otros? Mujeres y feminismo en los Programas universitarios de Historia y Filosofía". En dicha oportunidad apostamos a una mesa "general", que justamente hacía foco en el lugar del saber y el currículum, es decir, en el análisis de la Universidad, la producción y uso de conocimiento. Sin embargo, el trabajo fue redireccionado a una mesa "específica" sobre género. En definitiva, corporizar esta experiencia redobla el sentido de estas reflexiones.
} 


\section{A modo de cierre}

Comenzamos este artículo con un diagnóstico: a pesar de la visibilidad creciente de las teorías feministas y de género y del impacto de las reflexiones sobre su abordaje y enseñanza, aún encontramos obstáculos y dificultades para la plena inclusión y transversalización de estas perspectivas. Por ello, realizamos un análisis de los programas de estudio de la carrera de Historia (FaHCE/UNLP) mostrando que desde 2016 los temas vinculados a las teorías feministas y de género comienzan a visibilizarse con mayor vigor, al tiempo que, en ciertos espacios curriculares optativos, estos temas se abordan de manera sistemática desde 2005.

Luego identificamos un currículum en disputa, es decir, una interpelación crítica que, partiendo de las teorías feministas y de género, tensiona sentidos, autores/as y contenidos de estudio. Dicha disputa se encuentra motorizada, en buena parte, por los sectores estudiantiles. Mostramos que la paridad de representatividad de autores y autoras, aun cuando resulte condición necesaria y no suficiente, todavía constituye un horizonte lejano, como quedó en evidencia a través de las estadísticas relevadas por la agrupación estudiantil referida, así como por nuestro estudio exploratorio sobre programas de Introducción a la Filosofía en universidades nacionales.

Finalmente, identificamos una ambivalencia dado que muchas veces, la "incorporación" de estos temas se realiza bajo la modalidad del tema o clase especial. Ello trae aparejados diversos problemas, que problematizamos mediante una reflexión sobre la necesidad de transversalizar las teorías y debates feministas y de género, evitando la sectorización/guetificación de las investigaciones.

Desde la Universidad, resulta necesario pensar estos interrogantes, como algunos de los desafíos que las teorías feministas nos traen al presente. Converge en cierto sentido con la invitación a des-pensarnos para seguir pensando los saberes aprendidos y enseñados, evocando el decir de Boaventura de Sousa Santos (2010, p. 11). Los análisis que hemos presentado aquí intentaron indagar en esas construcciones que derivaron en matrices culturales de conocimiento, en el canon de la ciencia y del discurso académico, no para desterrarlo sino para 
estudiar sin perder de vista desde dónde miramos y hacemos ciencia, abonando en este sentido a una territorialidad de la profesión como investigadoras y docentes. En suma, apostar a una justicia curricular (CONNELL, 2009) que transforme el carácter patriarcal y eurocéntrico que todavía impera en las universidades.

\section{Referências}

ABATE, Stella y ORELLANO, Verónica. Notas sobre el currículum universitario: prácticas profesionales y saberes en uso:dossier. RevistaTrayectorias Universitarias, 15 oct. 2015. Disponible en:

https://revistas.unlp.edu.ar/TrayectoriasUniversitarias/article/view/2307/2252Acc eso en: 24 jul. 2020

AMORÓS, Celia. Hacia una crítica de la razón patriarcal. Barcelona: Anthropos, 1991.

ARGENTINA. Ley $n^{\circ}$ 26.150. Programa nacional de educación sexual integral, promulgada en octubre 2006. Buenos Aires: Congreso Argentino, 2006. Disponible en: http://servicios.infoleg.gob.ar/infoleglnternet/anexos/120000124999/121222/norma.htm. Acceso en: 12 jul. 2019.

BARZANI, Carlos A. (comp.). Actualidad de erotismo y pornografía. Buenos Aires: Topía, 2015.

BOLLA, Luisina. Sobre la teoría y la práctica feminista: una aproximación al feminismo materialista. In: JORNADAS DE SOCIOLOGÍA DE LA UNLP, 9., 2016.

Actas [...].Buenos Aires: UNLP, 2016. Disponible en:http://jornadassociologia.fahce.unlp.edu.ar/ix-jornadas/actas2016/PONmesa35Bolla.pdf. Acceso en: 12 jul. 2019.

CASTRO GÓMEZ, Santiago. Decolonizar la universidad:la hybris del punto cero y el diálogo de saberes. In: PALERMO, Zulma. Des/colonizar la universidad. Buenos Aires: Del Signo, 2015.

CISNE, Mirla. Feminismo materialista: uma análise marxista para além do gênero. In: MONTE ROCHA, Marcos Antonio (coord.). Feminismos plurais. Fortaleza: Expressão Gráfica e Editora, 2016. 
CONNELL, Robert William. La justicia curricular. LPP - Laboratorio de Políticas

Públicas, Buenos Aires, Año 6, n. 27, jul. 2009. Disponible en:

http://bibliotecavirtual.clacso.org.ar/Argentina/lpp/20100324023229/10.pdf.

Acceso en: 24 jul. 2020.

COSCARELLI, Raquel. Currículo universitario y formación docente. In: MORANDI, Glenda; UNGARO, Ana (comps). La experiencia interpelada: prácticas y

perspectivas en la formación docente universitaria. La Plata: EDULP, 2014.p. 95108.

COUDANNES AGUIRRE, Mariela. La formación del profesor de historia en la universidad argentina. La creciente distancia entre investigación/ docencia y teoría/ práctica. Revista Antíteses, v. 3,n. 6, p. 975-990, 2010. Disponible en:http://www.uel.br/revistas/uel/index.php/antiteses/article/view/4579. Acceso en: 24 jul. 2020

D 'ANTONIO, Débora. Género y clase: una mirada desde la historia social. REMS, Año 5-6, n. 5-6, nov. 2012-2013. Disponible en:http://estudiosmaritimossociales.org/wp-content/uploads/2014/01/remsnc2ba-5-6-dossier-gc3a9nero-y-clase-presentacic3b3n-d-antonio-1.pdf. Acceso en: 24 jul.2020

DAVINI, María Cristina. El currículo de formación del magisterio en la Argentina: planes de estudio y programas de enseñanza. Novedades educativas, n. 19, 1998. Disponible en:https://cdn.educ.ar/dinamico/UnidadHtml_get_2699e6d27a08-11e1-83d9-ed15e3c494af/Curriculum_Davini.pdf. Acceso en: 24 jul. 2020

DE ALBA, Alicia. Crisis, mito y perspectivas. Buenos Aires: Miño y Dávila, 1998.

DE LAURETIS, Teresa. Diferencias: etapas de un camino a través del feminismo. Madrid: Horas y horas, 2000.

FELDMAN, Daniel. Didáctica general: aportes para el desarrollo curricular. Buenos Aires: Ministerio de Educación de la Nación, 2010. Disponible en:http://repositorio.educacion.gov.ar:8080/dspace/bitstream/handle/123456789 /89818/Didactica\%20general.pdf?sequence=1. Acceso en: 24 jul. 2020.

ELIZALDE, Silvia; FELITTI Karina y QUEIROLO, Graciela. Género y sexualidades en las tramas del saber: revisiones y propuestas. Buenos Aires: Libros del Zorzal, 2009.

FEMENÍAS, María Luisa. ¿Qué le dan el feminismo y la teoría de género a la filosofía y su enseñanza?. In: CERLETTI, Alejandro y COULÓ, Ana (orgs.) La enseñanza filosófica: cuestiones de política, género y educación.Buenos Aires: Noveduc, 2015. 
FEMENÍAS, María Luisa. Género y feminismo en América Latina. Debate Feminista, año 20, n. 40, p. 42-74, 2009.

FOX KELLER, Evelyn. Reflexiones sobre género y ciencia. Valencia: Edicions Alfons El Magnànim, 1991.

HARAWAY, Donna. Situated knowledges: the science question in feminism and the privilege of partial perspective. Feminist Studies, v. 14,n. 3, p. 575-599, 1988.

HARAWAY, Donna. Ciencias, cyborgs y mujere: la invención de la naturaleza. Madrid: Sudakuir: Proletrario, 1995.

HARDING, Sandra. Ciencia y feminismo. Madrid: Morata, 1996.

LOPES LOURO, Guacira. Currículo, género y sexualidad: Lo "normal", lo "diferente" y lo "excéntrico". Descentrada, v. 3,n. 1, 2019. Disponible en: http://www.memoria.fahce.unlp.edu.ar/art_revistas/pr.9640/pr.9640.pdf. Acceso en: 24 jul. 2020.

MAFFÍA, Diana. Epistemología feminista: la subversión semiótica de las mujeres en la ciencia. Revista venezolana de estudios de la mujer, v. 12, n. 28, p. 63-98, 2007.

MATHIEU, Nicole-Claude. Critiques épistémologiques de la problématique des sexes dans le discours ethno-anthropologique. In: L'ANATOMIE POLITIQUE.

Donnemarie: iXe, 2013.

MORGADE, Graciela. Las Universidades Públicas como territorios del patriarcado. Política Universitaria: La universidad hoy, a 100 años de la Reforma. [Buenos Aires]: Instituto de Estudios y capacitación Federación Nacional de Docentes Universitarios, v. 1, mayo 2018.Especial. Disponible en:https://conadu.org.ar/wp-content/uploads/5-ESPECIAL-WEB.pdf. Acceso en: 24 jul. 2020.

PROGRAMA. Introducción a teoría feminista, estudios de género y sexualidades, La Plata: Facultad de Humanidades y Ciencias de la Educación, Universidad Nacional de La Plata, 2017.

ROCHA, Milagros. Mujeres y currículum en la formación universitaria: acerca del profesorado en historia de la Universidad Nacional de La Plata (1993-2017). 2019. Tesis (Maestría en Educación) - Facultad de Humanidades y Ciencias de la Educación, Universidad Nacional de La Plata, 2019. 
SILVA, Tomaz T. Documentos de identidad: una introducción a las teorías del currículo. Belo Horizonte: Autêntica Editorial, 1999. Disponible en:https://www.fceia.unr.edu.ar/geii/maestria/DoraBibliografia/Ut.\%201/SILVA\%2 Odocs\%20ident.pdf. Acceso en: 24 de jul. 2020.

SOUSA SANTOS, Boaventura de. Descolonizar el saber, reinventar el poder. Montevideo: Trilce: Universidad de la República, 2010. 